\title{
Antifibrotic Therapy in Chronic Hepatitis C
}

\author{
Rinaldo Focaccia Siciliano and Antonio Alci Barone \\ Laboratory of Hepatitis, LIM 47 DMIP-HC-FMUSP; São Paulo, SP, Brazil
}

The most pronounced histological characteristics of chronic hepatitis $\mathrm{C}$ are hepatocellular necrosis, inflammatory infiltrate and fibrosis. Unlike the first two, which present fluctuations, the evolution of liver fibrosis progressively evolves toward pathological sequelae due to the chronic liver injury induced by hepatitis $\mathrm{C}$ virus (HCV). Fibrosis results from the accumulation of extracellular matrix components, which leads to distortion of the liver architecture, alterations in hepatic microcirculation, and cellular dysfunction. This pathological hepatic process develops slowly and progressively, producing clinical repercussions only in its final stage, liver cirrhosis, which can take decades. Therefore, determination of the degree of fibrosis through liver biopsy or progression rate is crucial to the understanding of the natural history of chronic hepatitis C.

Some authors have identified host factors that are associated with the accelerated progression of liver fibrosis in HCV infection. The most important are being over 40 years of age at the time of HCV infection, being male, consuming excessive quantities of alcohol, and presenting conditions that lead to immunodeficiency, such as HIV infection or transplant. Other factors also seem to contribute to a more rapid progression toward fibrosis, such as liver steatosis, Coinfection with hepatitis B virus (HBV), obesity, and diabetes mellitus.

Recent advances in clinical studies and basic science have brought new perspectives to the development of therapies that can curb the progression of the fibrogenic process or even promote reversion of liver fibrosis. These advances occurred after the recognition of fibrogenic cell types in the liver, as well as of the principal sites of fibrosis formation and its reversibility potential.

Fibrosis seems to result from an imbalance between the synthesis and degradation of the extracellular matrix, resulting in the accumulation of conjunctive tissue in the liver. This process is triggered and sustained by the chronic liver damage caused by HCV and leads to the disruption of the normal liver architecture, culminating in the development of cirrhosis/ hepatic insufficiency. Stellate cells are the principal source of extracellular matrix in the damaged liver tissue. In a normal liver, they are present in the Disse space and are the principal reservoirs of vitamin A. Chronic hepatic injury secondary to $\mathrm{HCV}$, through the increase of free radicals and fibrogenic mediators, leads to the activation of stellate cells, as well as to their proliferation and differentiation into myofibroblasts, when they acquire contractile, pro-inflammatory and fibrogenic properties. Once activated, they migrate to the sites of injury

The Brazilian Journal of Infectious Diseases 2007;11 (5) Suppl. 1:20-21. (C) 2007 by The Brazilian Journal of Infectious Diseases and Contexto Publishing. All rights reserved. with the objective of effecting repair by secreting a large quantity of extracellular matrix. Activated stellate cells also produce cytokines that perpetuate their activation status, as well as being stimulated by the apoptosis of damaged hepatocytes. The leukocytes attracted into the hepatic inflammatory process secondary to $\mathrm{HCV}$ infection also induce the production of collagen by stellate cells. These activated cells, in turn, have a pro-inflammatory effect that feeds a vicious cycle.

Under physiological conditions, the excess extracellular matrix is degraded through regulation by metalloproteinases. Activated hepatic myofibroblasts, in addition to producing large quantities of Type I and Type III collagen, secrete tissue inhibitors of metalloproteinases, which block the collagenolytic activity. We have begun to comprehend various parts of this process of accumulation of extracellular matrix in the liver tissue, although there are still many gaps in our understanding. In addition to the efficient collagenolytic activity of the metalloproteinases, the degradation of the extracellular matrix in the liver can also occur through the activities of neutrophils, macrophages and stellate cells themselves; however, the importance and modulation of each have yet to be clarified. The activation of stellate cells, as well as their proliferation and fibrogenic activity, is regulated by various soluble growth factors, such as platelet-derived growth factor, transformation growth factor beta, and endothelin-1.

Determining the mechanisms involved in the process of liver fibrosis has led to a new perspective on the development of antifibrotic drugs in animal models. However, there is still an obstacle to be transposed before positive laboratory results can be reproduced in humans. New lines of research attempt to attenuate the activation of stellate cells, inhibit some of their properties when activated, promote their apoptosis, or stimulate the degradation of the extracellular matrix. No specific antifibrotic therapies have been approved for use in humans; however, considerable effort has been made in laboratory studies in this promising area.

In clinical practice, the treatment of hepatitis $\mathrm{C}$ with the combination of pegylated interferon alpha and ribavirin is the only pharmacological regimen currently available that can modify the natural evolution of liver fibrosis. This does not simply occur by suppressing the viremia (achieving a sustained virological response) but possibly by the antifibrotic role of pegylated interferon alpha. Recent clinical studies show remission of liver fibrosis or reduction of its progression rate in patients under treatment with therapeutic regimens containing interferon alpha or pegylated interferon. This effect is even more apparent among hepatitis $\mathrm{C}$ patients presenting a biochemical or virological response after therapy. Notably, 
most of these studies are retrospective, and their principal objective was the evaluation of the virologic response to the treatment with interferon alpha.

Although liver fibrosis is a dynamic process, its remission is slow and demands prolonged therapy and follow-up evaluation. Multicentric prospective comparative studies that evaluate the histological impact of maintenance treatment with pegylated interferon in low doses versus placebo in patients with hepatitis $\mathrm{C}$ who are nonresponsive to treatment are currently being carried out. Preliminary results of these studies are quite promising; reduction of fibrosis or clinical complications resulting from liver cirrhosis can be seen after approximately two years of therapy with pegylated interferon. The final results, with histological analysis, after long-term follow-up treatment and inclusion of a large number of patients, will consolidate an evaluation of safety, clinical and histological benefits, and cost-effectiveness ratio of the prolonged use of pegylated interferon in low doses in hepatitis $\mathrm{C}$.

The role of the physician in the positive modification of the natural history of hepatitis C-related liver fibrosis should not be restricted to the removal of $\mathrm{HCV}$ as a hepatic aggressive/ pro-inflammatory factor (achieving a sustained virologic response) or to the use of interferon as a potential antifibrotic agent. In daily practice, other strategies aimed at attenuating the progression of liver fibrosis are based on the effects on modifiable factors that can alter the natural history of HCV: drinking cessation; prevention and control of obesity; and prevention of HIV or HBV co-infection.

Much progress has been achieved in basic science regarding the understanding of the biological mechanisms that lead to the development of liver fibrosis. However, clinical trials to validate new drugs or antifibrotic strategies are warranted.

\section{References}

1. Albanis E, Friedman S,L. Antifibrotic agents for liver disease. Am J Transplant 2006;6(1):12-9.

2. Friedman S.L., Rockey D.C., Bissell D.M. Hepatic fibrosis 2006: report of the Third AASLD Single Topic Conference. Hepatology 2007;45(1):242-9.

3. Bataller R., Brenner D.A. Liver fibrosis. J Clin Invest 2005;115(2):209-18.

4. Marcellin P., Asselah T., Boyer N. Fibrosis and disease progression in hepatitis C. Hepatology 2002;36(5 Suppl 1):S47-56.

5. Friedman S.L., Bansal M.B. Reversal of hepatic fibrosis - fact or fantasy? Hepatology 2006;43(2 Suppl 1):S82-8.

6. Everson G.T., Hoefs J.C., Seeff L.B., et al. Impact of disease severity on outcome of antiviral therapy for chronic hepatitis C: Lessons from the HALT-C trial. Hepatology 2006;44(6):1675-84.

7. Kaiser P., Hass H., Lutze B., et al. Long-term low dose treatment with pegylated interferon alpha $2 \mathrm{~b}$ leads to a significant reduction in fibrosis and inflammatory score in chronic hepatitis $\mathrm{C}$ nonresponder patients with fibrosis or cirrhosis. 57th Annual Meeting of the American Association for the Study of Liver Diseases. Massachusetts - USA 2006.

8. Afdhal N., Freilich B., Levine R., et al. Colchicine versus peginterferon long-term (COPILOT) trial: interim analysis of clinical outcomes at year 2. Hepatology 2004;40:238A. 